DOI: $10.15393 /$ j3.art.2022.10550

UDC 517.54

A. Alb LupAŞ

\title{
SUBORDINATION RESULTS FOR A FRACTIONAL INTEGRAL OPERATOR
}

\begin{abstract}
In this paper, we establish several differential subordinations regarding the operator $D_{z}^{-\lambda} S R^{m, n}$ defined using the fractional integral of the differential operator $S R^{m, n}$, obtained as a convolution product of Sălăgean operator $S^{m}$ and Ruscheweyh derivative $R^{n}$. By means of the newly obtained operator, a new subclass of analytic functions denoted by $\mathcal{S R}_{m, n, \lambda}(\delta)$ is introduced and various properties and characteristics of this class are derived, making use of the concept of differential subordination.
\end{abstract}

Key words: analytic function, differential subordination, fractional integral, convolution product, Sălăgean operator, Ruscheweyh derivative

2020 Mathematical Subject Classification: $30 C 45,34 A 40$

1. Introduction. Denote by $U$ the unit disc of the complex plane, $U=\{z \in \mathbb{C}:|z|<1\}$ and by $\mathcal{H}(U)$ the space of holomorphic functions in $U$.

Let $\mathcal{A}(p, l)=\left\{f \in \mathcal{H}(U): f(z)=z^{p}+\sum_{j=p+l}^{\infty} a_{j} z^{j}, z \in U\right\}, \mathcal{A}(1, l)=\mathcal{A}_{l}$ and $\mathcal{H}[a, l]=\left\{f \in \mathcal{H}(U): f(z)=a+a_{l} z^{l}+a_{l+1} z^{l+1}+\ldots, z \in U\right\}$, where $p, l \in \mathbb{N}, a \in \mathbb{C}$.

The well-known definitions for Sălăgean and Ruscheweyh operators and the convolution product of these operators are also reminded:

Definition 1. (Sălăgean [11]) For $f \in \mathcal{A}_{l}$, and $n \in \mathbb{N}$, the operator $S^{n}$ is defined by $S^{n}: \mathcal{A}_{l} \rightarrow \mathcal{A}_{l}$,

$$
\begin{gathered}
S^{0} f(z)=f(z), \\
S^{1} f(z)=z f^{\prime}(z), \\
\cdots \cdots \cdots \cdots \cdots \\
S^{n+1} f(z)=z\left(S^{n} f(z)\right)^{\prime}, \quad z \in U .
\end{gathered}
$$

(C) Petrozavodsk State University, 2022 
Remark 1. If $f \in \mathcal{A}_{l}, f(z)=z+\sum_{j=l+1}^{\infty} a_{j} z^{j}$, then $S^{n} f(z)=z+\sum_{j=l+1}^{\infty} j^{n} a_{j} z^{j}$, $z \in U$.

Definition 2. (Ruscheweyh [10]) For $f \in \mathcal{A}_{l}$ and $n \in \mathbb{N}$, the operator $R^{n}$ is defined by $R^{n}: \mathcal{A}_{l} \rightarrow \mathcal{A}_{l}$,

$$
\begin{aligned}
& R^{0} f(z)=f(z), \\
& R^{1} f(z)=z f^{\prime}(z), \\
& \cdots \cdots \cdots \cdots \cdots \\
(n+1) R^{n+1} f(z)= & z\left(R^{n} f(z)\right)^{\prime}+n R^{n} f(z), \quad z \in U .
\end{aligned}
$$

Remark 2. If $f \in \mathcal{A}_{l}, f(z)=z+\sum_{j=l+1}^{\infty} a_{j} z^{j}$, then

$$
R^{n} f(z)=z+\sum_{j=l+1}^{\infty} \frac{\Gamma(n+j)}{\Gamma(n+1) \Gamma(j)} a_{j} z^{j}, \quad z \in U,
$$

where $\Gamma$ is the gamma function.

Let $f, g \in \mathcal{A}$, where $f$ and $g$ are defined by $f(z)=z+\sum_{j=2}^{\infty} a_{j} z^{j}$ and $g(z)=z+\sum_{j=2}^{\infty} b_{j} z^{j}$. Then the Hadamard product (or convolution) $f * g$ of the functions $f$ and $g$ is defined by

$$
(f * g)(z)=z+\sum_{j=2}^{\infty} a_{j} b_{j} z^{j} .
$$

Definition 3. [1] Let $n, m \in \mathbb{N}$. Denote by $S R^{m, n}: \mathcal{A}_{l} \rightarrow \mathcal{A}_{l}$ the operator given by the Hadamard product of the Sălăgean operator $S^{m}$ and the Ruscheweyh derivative $R^{n}$,

$$
S R^{m, n} f(z)=\left(S^{m} * R^{n}\right) f(z),
$$

for any $z \in U$.

Remark 3. [1] If $f \in \mathcal{A}_{l}$ and $f(z)=z+\sum_{j=l+1}^{\infty} a_{j} z^{j}$, then

$$
S R^{m, n} f(z)=z+\sum_{j=l+1}^{\infty} j^{m} \frac{\Gamma(n+j)}{\Gamma(n+1) \Gamma(j)} a_{j}^{2} z^{j}, \quad z \in U .
$$


Definition 4. [7] The fractional integral of order $\lambda(\lambda>0)$ is defined for a function $f$ by

$$
D_{z}^{-\lambda} f(z)=\frac{1}{\Gamma(\lambda)} \int_{0}^{z} \frac{f(t)}{(z-t)^{1-\lambda}} d t,
$$

where $f$ is an analytic function in a simply-connected region of the $z$ plane containing the origin, and the multiplicity of $(z-t)^{\lambda-1}$ is removed by requiring $\log (z-t)$ to be real, when $(z-t)>0$.

The fractional integral is a function used intensely for obtaining new operators that generate interesting subclasses of functions, providing useful and inspiring outcome related to them [2-6]. Similar methods are used in the present investigation to obtain results contained in the next section.

Using Definition 3 and Definition 4, we get the fractional integral associated with the differential operator $S R^{m, n}$. Using this operator, a new subclass of analytic functions is introduced and investigated using the methods of the theory of differential subordinations.

Two lemmas useful for proving the original results of the paper are now given.

Lemma 1. (Miller and Mocanu [9]) Let $g$ be a convex function in $U$ and let $h(z)=g(z)+\operatorname{l\alpha } z g^{\prime}(z)$, for $z \in U$, where $\alpha>0$ and $l$ be a positive integer.

$$
\begin{gathered}
\text { If } p(z)=g(0)+p_{l} z^{l}+p_{l+1} z^{l+1}+\cdots, z \in U, \text { is holomorphic in } U \text { and } \\
p(z)+\alpha z p^{\prime}(z) \prec h(z), \quad z \in U,
\end{gathered}
$$

then

$$
p(z) \prec g(z), \quad z \in U,
$$

and this result is sharp.

Lemma 2. (Hallenbeck and Ruscheweyh [8]) Let $h$ be a convex function with $h(0)=a$, and let $\gamma \in \mathbb{C} \backslash\{0\}$ be a complex number with $\operatorname{Re} \gamma \geqslant 0$. If $p \in \mathcal{H}[a, l]$ and

$$
p(z)+\frac{1}{\gamma} z p^{\prime}(z) \prec h(z), \quad \text { for } \quad z \in U,
$$

then

$$
p(z) \prec g(z) \prec h(z), \quad \text { for } \quad z \in U \text {, }
$$


where $g(z)=\frac{\gamma}{l z^{\gamma / l}} \int_{0}^{z} h(t) t^{\gamma / l-1} d t$, for $z \in U$.

2. Main results. We introduce the fractional integral associated with the differential operator $S R^{m, n}$.

Definition 5. Let $\lambda>0$ and $m, n \in \mathbb{N}$. The fractional integral associated with the linear differential operator $S R^{m, n} f$ is defined by

$$
\begin{gathered}
D_{z}^{-\lambda} S R^{m, n} f(z)=\frac{1}{\Gamma(\lambda)} \int_{0}^{z} \frac{S R^{m, n} f(t)}{(z-t)^{1-\lambda}} d t= \\
=\frac{1}{\Gamma(\lambda)}\left(\int_{0}^{z} \frac{t}{(z-t)^{1-\lambda}} d t+\sum_{j=l+1}^{\infty}\left(j^{m} \frac{\Gamma(n+j)}{\Gamma(n+1) \Gamma(j)}\right) a_{j}^{2} \int_{0}^{z} \frac{t^{j}}{(z-t)^{1-\lambda}} d t\right),
\end{gathered}
$$

which is written in the following form after a simple calculation:

$$
D_{z}^{-\lambda} S R^{m, n} f(z)=\frac{1}{\Gamma(\lambda+2)} z^{\lambda+1}+\sum_{j=l+1}^{\infty} \frac{j^{m+1} \Gamma(n+j)}{\Gamma(n+1) \Gamma(j+\lambda+1)} a_{j}^{2} z^{j+\lambda},
$$

for $f(z)=z+\sum_{j=l+1}^{\infty} a_{j} z^{j} \in \mathcal{A}_{l}$. Note that $D_{z}^{-\lambda} S R^{m, n} f(z) \in \mathcal{A}(\lambda+1, l)$.

Another simple calculation gives the relation

$$
z\left(D_{z}^{-\lambda} S R^{m, n} f(z)\right)^{\prime}=\lambda D_{z}^{-\lambda} S R^{m, n} f(z)+D_{z}^{-\lambda} S R^{m+1, n} f(z), \quad z \in U .
$$

Firstly, we define and study a subclass of analytic functions using the differential subordinations regarding to the differential operator $D_{z}^{-\lambda} S R^{m, n}: \mathcal{A}_{l} \rightarrow \mathcal{A}_{l}$.

Definition 6. Let $\delta \in[0,1)$ and $m, n \in \mathbb{N}, \lambda>0$. A function $f \in \mathcal{A}_{l}$ is said to be in the class $\mathcal{S} \mathcal{R}_{m, n, \lambda}(\delta)$ if it satisfies the inequality

$$
\operatorname{Re}\left(D_{z}^{-\lambda} S R^{m, n} f(z)\right)^{\prime}>\delta, \quad z \in U .
$$

Theorem 1. Let $g$ be a convex function in $U, h(z)=g(z)+\frac{1}{c+2} z g^{\prime}(z)$, $z \in U$, where $c>0$. If $f \in \mathcal{S R}_{m, n, \lambda}(\delta)$ and

$$
F(z)=I_{c}(f)(z)=\frac{c+2}{z^{c+1}} \int_{0}^{z} t^{c} f(t) d t, \quad z \in U
$$


then

$$
\left(D_{z}^{-\lambda} S R^{m, n} f(z)\right)^{\prime} \prec h(z), \quad z \in U
$$

implies

$$
\left(D_{z}^{-\lambda} S R^{m, n} F(z)\right)^{\prime} \prec g(z), \quad z \in U,
$$

and this result is sharp.

Proof. We have $z^{c+1} F(z)=(c+2) \int_{0}^{z} t^{c} f(t) d t$. Differentiating with respect to $z$, we obtain

$$
(c+1) F(z)+z F^{\prime}(z)=(c+2) f(z)
$$

and

$$
\begin{aligned}
& (c+1) D_{z}^{-\lambda} S R^{m, n} F(z)+z\left(D_{z}^{-\lambda} S R^{m, n} F(z)\right)^{\prime}=(c+2) D_{z}^{-\lambda} S R^{m, n} f(z), \\
& z \in U .
\end{aligned}
$$

Differentiating (7), we get

$$
\left(D_{z}^{-\lambda} S R^{m, n} F(z)\right)^{\prime}+\frac{1}{c+2} z\left(D_{z}^{-\lambda} S R^{m, n} F(z)\right)^{\prime \prime}=\left(D_{z}^{-\lambda} S R^{m, n} f(z)\right)^{\prime},
$$

$z \in U$.

Using (8), the differential subordination (5) becomes

$$
\left(D_{z}^{-\lambda} S R^{m, n} F(z)\right)^{\prime}+\frac{1}{c+2} z\left(D_{z}^{-\lambda} S R^{m, n} F(z)\right)^{\prime \prime} \prec g(z)+\frac{1}{c+2} z g^{\prime}(z) .
$$

If we denote

$$
p(z)=\left(D_{z}^{-\lambda} S R^{m, n} F(z)\right)^{\prime}
$$

then $p \in \mathcal{H}[\lambda+1, l]$.

Replacing (10) in (9), we obtain

$$
p(z)+\frac{1}{c+2} z p^{\prime}(z) \prec g(z)+\frac{1}{c+2} z g^{\prime}(z), \quad z \in U .
$$

Using Lemma 1, we have

$$
p(z) \prec g(z) \quad \text { i.e. } \quad\left(D_{z}^{-\lambda} S R^{m, n} F(z)\right)^{\prime} \prec g(z), \quad z \in U,
$$

and $g$ is the best dominant. 
Theorem 2. Let $h(z)=\frac{1+(2 \delta-1) z}{1+z}, \delta \in[0,1)$ and $c>0$. If $m, n \in \mathbb{N}$, $\lambda>0$, and $I_{c}$ is given by Theorem 1 , then

$$
I_{c}\left[\mathcal{S R}_{m, n, \lambda}(\delta)\right] \subset \mathcal{S} \mathcal{R}_{m, n, \lambda}\left(\delta^{*}\right),
$$

where $\delta^{*}=2 \delta-1+\frac{(c+2)(2-2 \delta)}{l} \beta\left(\frac{c+2}{l}-2\right)$ and $\beta(x)=\int_{0}^{1} \frac{t^{x+1}}{t+1} d t$.

Proof. The function $h$ is convex; using the same steps as in the proof of Theorem 1, we get, from the hypothesis of Theorem 2, the following:

$$
p(z)+\frac{1}{c+2} z p^{\prime}(z) \prec h(z),
$$

where $p(z)$ is defined in (10).

Using Lemma 2, we deduce that

$$
p(z) \prec g(z) \prec h(z),
$$

that is

$$
\left(D_{z}^{-\lambda} S R^{m, n} F(z)\right)^{\prime} \prec g(z) \prec h(z),
$$

where

$$
\begin{aligned}
& g(z)=\frac{c+2}{l z^{\frac{c+2}{l}}} \int_{0}^{z} t^{\frac{c+2}{l}-1} \frac{1+(2 \delta-1) t}{1+t} d t \\
&=(2 \delta-1)+\frac{(c+2)(2-2 \delta)}{l z^{\frac{c+2}{l}}} \int_{0}^{z} \frac{t^{\frac{c+2}{l}-1}}{1+t} d t .
\end{aligned}
$$

Since $g$ is convex and $g(U)$ is symmetric with respect to the real axis, we deduce

$$
\begin{gathered}
\operatorname{Re}\left(D_{z}^{-\lambda} S R^{m, n} F(z)\right)^{\prime} \geqslant \min _{|z|=1} \operatorname{Re} g(z)=\operatorname{Re} g(1)=\delta^{*}= \\
=2 \delta-1+\frac{(c+2)(2-2 \delta)}{l} \beta\left(\frac{c+2}{l}-2\right) .
\end{gathered}
$$

From (12), we deduce the subordination (11).

Theorem 3. Let $g$ be a convex function, $g(0)=0$, and let $h$ be the function $h(z)=g(z)+z g^{\prime}(z), z \in U$. If $m, n \in \mathbb{N}, f \in \mathcal{A}_{l}$ and follows the differential subordination

$$
\left(D_{z}^{-\lambda} S R^{m, n} f(z)\right)^{\prime} \prec h(z) \quad z \in U,
$$


then

$$
\frac{D_{z}^{-\lambda} S R^{m, n} f(z)}{z} \prec g(z), \quad z \in U,
$$

and this result is sharp.

Proof. For $f \in \mathcal{A}_{l}, f(z)=z+\sum_{j=l+1}^{\infty} a_{j} z^{j}$, we have

$$
D_{z}^{-\lambda} S R^{m, n} f(z)=\frac{1}{\Gamma(\lambda+2)} z^{\lambda+1}+\sum_{j=l+1}^{\infty} \frac{j^{m+1} \Gamma(n+j)}{\Gamma(n+1) \Gamma(j+\lambda+1)} a_{j}^{2} z^{j+\lambda},
$$

$z \in U$.

Consider

$$
\begin{gathered}
p(z)=\frac{D_{z}^{-\lambda} S R^{m, n} f(z)}{z}= \\
=\frac{\frac{1}{\Gamma(\lambda+2)} z^{\lambda+1}+\sum_{j=l+1}^{\infty} \frac{j^{m+1} \Gamma(n+j)}{\Gamma(n+1) \Gamma(j+\lambda+1)} a_{j}^{2} z^{j+\lambda}}{z}= \\
=\frac{1}{\Gamma(\lambda+2)} z^{\lambda}+\sum_{j=l+1}^{\infty} \frac{j^{m+1} \Gamma(n+j)}{\Gamma(n+1) \Gamma(j+\lambda+1)} a_{j}^{2} z^{j+\lambda-1}, p \in \mathcal{H}[0, \lambda] .
\end{gathered}
$$

We have

$$
p(z)+z p^{\prime}(z)=\left(D_{z}^{-\lambda} S R^{m, n} f(z)\right)^{\prime}, \quad z \in U .
$$

Then

$$
\left(D_{z}^{-\lambda} S R^{m, n} f(z)\right)^{\prime} \prec h(z), \quad z \in U
$$

becomes

$$
p(z)+z p^{\prime}(z) \prec h(z)=g(z)+z g^{\prime}(z), \quad z \in U .
$$

By using Lemma 1, we obtain

$$
p(z) \prec g(z), \quad z \in U \text {, i. e. } \frac{D_{z}^{-\lambda} S R^{m, n} f(z)}{z} \prec g(z), \quad z \in U \text {. }
$$

Theorem 4. Let $h \in \mathcal{H}(U)$, with $h(0)=0$, which satisfies the inequality $\operatorname{Re}\left(1+\frac{z h^{\prime \prime}(z)}{h^{\prime}(z)}\right)>-\frac{1}{2}, z \in U$. If $m, n \in \mathbb{N}, \lambda>0, f \in \mathcal{A}_{l}$ and is subject to the differential subordination

$$
\left(D_{z}^{-\lambda} S R^{m, n} f(z)\right)^{\prime} \prec h(z), \quad z \in U ;
$$


then

$$
\frac{D_{z}^{-\lambda} S R^{m, n} f(z)}{z} \prec q(z), \quad z \in U,
$$

where $q(z)=\frac{1}{l z^{\frac{1}{l}}} \int_{0}^{z} h(t) t^{\frac{1}{l}-1} d t$. The function $q$ is convex, and it is the best dominant.

Proof. Let

$$
\begin{gathered}
p(z)=\frac{D_{z}^{-\lambda} S R^{m, n} f(z)}{z}= \\
=\frac{1}{\Gamma(\lambda+2)} z^{\lambda}+\sum_{j=l+1}^{\infty} \frac{j^{m+1} \Gamma(n+j)}{\Gamma(n+1) \Gamma(j+\lambda+1)} a_{j}^{2} z^{j+\lambda-1},
\end{gathered}
$$

$z \in U, p \in \mathcal{H}[0, \lambda]$.

Differentiating, we obtain

$$
\left(D_{z}^{-\lambda} S R^{m, n} f(z)\right)^{\prime}=p(z)+z p^{\prime}(z), \quad z \in U,
$$

and (14) becomes

$$
p(z)+z p^{\prime}(z) \prec h(z), \quad z \in U .
$$

Using Lemma 2, we have

$$
p(z) \prec q(z)=\frac{1}{l z^{\frac{1}{l}}} \int_{0}^{z} h(t) t^{\frac{1}{l}-1} d t, \quad z \in U,
$$

i.e.,

$$
\frac{D_{z}^{-\lambda} S R^{m, n} f(z)}{z} \prec q(z)=\frac{1}{l z^{\frac{1}{l}}} \int_{0}^{z} h(t) t^{\frac{1}{l}-1} d t, \quad z \in U,
$$

and $q$ is the best dominant.

Corollary 1 . Let $h(z)=\frac{(2 \beta-1) z}{1+z}$ be a convex function in $U, 0 \leqslant \beta<1$. If $m, n \in \mathbb{N}, \lambda>0, f \in \mathcal{A}_{l}$ and satisfies the differential subordination

$$
\left(D_{z}^{-\lambda} S R^{m, n} f(z)\right)^{\prime} \prec h(z), \quad z \in U,
$$

then

$$
\frac{D_{z}^{-\lambda} S R^{m, n}(z)}{z} \prec q(z), \quad z \in U,
$$


where $q$ is given by $q(z)=\frac{2 \beta-1}{l z^{\frac{1}{l}}} \int_{0}^{z} \frac{t^{\frac{1}{l}}}{1+t} d t, z \in U$. The function $q$ is convex and it is the best dominant.

Proof. Following the same steps as in the proof of Theorem 4 and considering $p(z)=\frac{D_{z}^{-\lambda} S R^{m, n} f(z)}{z}$, the differential subordination (15) is transformed to

$$
p(z)+z p^{\prime}(z) \prec h(z)=\frac{(2 \beta-1) z}{1+z}, \quad z \in U .
$$

Using Lemma 2 for $\gamma=1$, we get $p(z) \prec q(z)$, i. e.,

$$
\begin{aligned}
\frac{D_{z}^{-\lambda} S R^{m, n} f(z)}{z} & \prec q(z)=\frac{1}{l z^{\frac{1}{l}}} \int_{0}^{z} h(t) t^{\frac{1}{l}-1} d t= \\
= & \frac{1}{l z^{\frac{1}{l}}} \int_{0}^{z} t^{\frac{1}{l}-1} \frac{(2 \beta-1) t}{1+t} d t=\frac{2 \beta-1}{l z^{\frac{1}{l}}} \int_{0}^{z} \frac{t^{\frac{1}{l}}}{1+t} d t, \quad z \in U .
\end{aligned}
$$

Theorem 5. Let $g$ be a convex function, such that $g(0)=0$, and let $h$ be the function $h(z)=g(z)+\frac{l}{1-\lambda} z g^{\prime}(z), z \in U, m, n \in \mathbb{N}, \lambda>0$. If $f \in \mathcal{A}_{l}$ and the differential subordination

$$
\frac{\lambda}{1-\lambda} \frac{D_{z}^{-\lambda} S R^{m+1, n} f(z)}{z}+\frac{1}{1-\lambda} \frac{D_{z}^{-\lambda} S R^{m+2, n} f(z)}{z} \prec h(z), \quad z \in U,
$$

holds, then

$$
\left(D_{z}^{-\lambda} S R^{m, n} f(z)\right)^{\prime} \prec g(z), \quad z \in U,
$$

and this result is sharp.

Proof. With notation

$p(z)=\left(D_{z}^{-\lambda} S R^{m, n} f(z)\right)^{\prime}=\frac{1}{\Gamma(\lambda+1)} z^{\lambda}+\sum_{j=l+1}^{\infty} \frac{j^{m+1} \Gamma(n+j)}{\Gamma(n+1) \Gamma(j+\lambda)} a_{j}^{2} z^{j+\lambda-1}$

and $p(0)=0$, we obtain for $f(z)=z+\sum_{j=l+1}^{\infty} a_{j} z^{j}$, taking account relation (3),

$$
p(z)+z p^{\prime}(z)=\lambda p(z)+\frac{\lambda D_{z}^{-\lambda} S R^{m+1, n} f(z)}{z}+\frac{D_{z}^{-\lambda} S R^{m+2, n} f(z)}{z} .
$$


We have

$$
p(z)+\frac{1}{1-\lambda} z p^{\prime}(z) \prec h(z)=g(z)+\frac{l}{1-\lambda} z g^{\prime}(z), \quad z \in U .
$$

Using Lemma 1, we obtain

$$
p(z) \prec g(z), \quad z \in U \text {, i.e. }\left(D_{z}^{-\lambda} S R^{m, n} f(z)\right)^{\prime} \prec g(z), \quad z \in U,
$$

and this result is sharp.

Theorem 6. Let $h \in \mathcal{H}(U)$ with $h(0)=0$, which satisfies the inequality $\operatorname{Re}\left[1+\frac{z h^{\prime \prime}(z)}{h^{\prime}(z)}\right]>-\frac{1}{2}, z \in U$. If $m, n \in \mathbb{N}, \lambda>0, f \in \mathcal{A}_{l}$ and satisfies the differential subordination

$$
\frac{\lambda}{1-\lambda} \frac{D_{z}^{-\lambda} S R^{m+1, n} f(z)}{z}+\frac{1}{1-\lambda} \frac{D_{z}^{-\lambda} S R^{m+2, n} f(z)}{z} \prec h(z), \quad z \in U,
$$

then

$$
\left(D_{z}^{-\lambda} S R^{m, n} f(z)\right)^{\prime} \prec q(z), \quad z \in U
$$

where $q$ is given by $q(z)=\frac{1-\lambda}{l z^{\frac{1-\lambda}{l}}} \int_{0}^{z} h(t) t^{\frac{1-\lambda}{l}-1} d t$. The function $q$ is convex and it is the best dominant.

Proof. Using the properties of operator $D_{z}^{-\lambda} S R^{m, n}$ and considering $p(z)=\left(D_{z}^{-\lambda} S R^{m, n} f(z)\right)^{\prime}$, we obtain

$$
\frac{\lambda}{1-\lambda} \frac{D_{z}^{-\lambda} S R^{m+1, n} f(z)}{z}+\frac{1}{1-\lambda} \frac{D_{z}^{-\lambda} S R^{m+2, n} f(z)}{z}=p(z)+\frac{1}{1-\lambda} z p^{\prime}(z),
$$

$z \in U$.

Then (17) becomes

$$
p(z)+\frac{1}{1-\lambda} z p^{\prime}(z) \prec h(z), \quad z \in U .
$$

Since $p \in \mathcal{H}[0, \lambda]$, using Lemma 2 for $\gamma=1-\lambda$, we deduce

$$
p(z) \prec q(z), \quad z \in U,
$$

where

$$
q(z)=\frac{1-\lambda}{l z^{\frac{1-\lambda}{l}}} \int_{0}^{z} h(t) t^{\frac{1-\lambda}{l}-1} d t, \quad z \in U
$$


i. e.,

$$
\left(D_{z}^{-\lambda} S R^{m, n} f(z)\right)^{\prime} \prec q(z)=\frac{1-\lambda}{l z^{\frac{1-\lambda}{l}}} \int_{0}^{z} h(t) t^{\frac{1-\lambda}{l}-1} d t, \quad z \in U,
$$

and $q$ is the best dominant.

Corollary 1. Let $h(z)=\frac{(2 \beta-1) z}{1+z}$ be a convex function in $U, 0 \leqslant \beta<1$. If $m, n \in \mathbb{N}, \lambda>0, f \in \mathcal{A}_{l}$ and satisfies the differential subordination

$$
\frac{\lambda}{1-\lambda} \frac{D_{z}^{-\lambda} S R^{m+1, n} f(z)}{z}+\frac{1}{1-\lambda} \frac{D_{z}^{-\lambda} S R^{m+2, n} f(z)}{z} \prec h(z), \quad z \in U,
$$

then

$$
\left(D_{z}^{-\lambda} S R^{m, n} f(z)\right)^{\prime} \prec q(z), \quad z \in U
$$

where $q$ is given by $q(z)=\frac{(1-\lambda)(2 \beta-1)}{l z^{\frac{1-\lambda}{l}}} \int_{0}^{z} \frac{t^{\frac{1-\lambda}{l}}}{1+t} d t, z \in U$. The function $q$ is convex and it is the best dominant.

Proof. Following the same steps as in the proof of Theorem 5 and considering $p(z)=\left(D_{z}^{-\lambda} S R^{m, n} f(z)\right)^{\prime}$, the differential subordination (18) is transformed to

$$
p(z)+\frac{1}{1-\lambda} z p^{\prime}(z) \prec h(z)=\frac{(2 \beta-1) z}{1+z}, \quad z \in U .
$$

Using Lemma 2 for $\gamma=1-\lambda$, we have $p(z) \prec q(z)$, i. e.,

$$
\begin{aligned}
& \left(D_{z}^{-\lambda} S R^{m, n} f(z)\right)^{\prime} \prec q(z)=\frac{1-\lambda}{l z^{\frac{1-\lambda}{l}}} \int_{0}^{z} h(t) t^{\frac{1-\lambda}{l}-1} d t= \\
& =\frac{1-\lambda}{l z^{\frac{1-\lambda}{l}}} \int_{0}^{z} t^{\frac{1-\lambda}{l}}-1 \frac{(2 \beta-1) t}{1+t} d t=\frac{(1-\lambda)(2 \beta-1)}{l z^{\frac{1-\lambda}{l}}} \int_{0}^{z} \frac{t^{\frac{1-\lambda}{l}}}{1+t} d t, \quad z \in U .
\end{aligned}
$$

\section{References}

[1] Alb-Lupaş A. A subclass of analytic functions defined by a fractional integral operator. J. Comput. Anal. Appl., 2019, vol. 27, no. 3, pp. 502-505. 
[2] Alb-Lupaş A. About a Subclass of Analytic Functions Defined by a Fractional Integral Operator. Montes Taurus J. Pure Appl. Math., 2021, vol. 3 (3), pp. $200-210$.

[3] Alb-Lupaş A. Properties on a subclass of analytic functions defined by a fractional integral operator. J. Comput. Anal. Appl., 2019, vol. 27, no. 3, pp. $506-510$.

[4] Alb-Lupaş A. New Applications of the Fractional Integral on Analytic Functions. Symmetry 2021, 13, 423.

DOI: https://doi.org/10.3390/sym13030423

[5] Alb-Lupaş A., Cătaş A. An Application of the Principle of Differential Subordination to Analytic Functions Involving Atangana-Baleanu Fractional Integral of Bessel Functions. Symmetry 2021, 13, 971.

DOI: https://doi.org/10.3390/sym13060971

[6] Alb-Lupaş A., Oros G. I. Differential Subordination and Superordination Results Using Fractional Integral of Confluent Hypergeometric Function. Symmetry 2021, 13, 327.

DOI: https://doi.org/10.3390/sym13020327

[7] Cho N. E., Aouf A. M. K. Some applications of fractional calculus operators to a certain subclass of analytic functions with negative coefficients. Tr. J. of Mathematics, 1996, vol. 20, pp. 553-562.

[8] Hallenbeck D. J., Ruscheweyh St. Subordination by convex functions. Proc. Amer. Math. Soc., 1975, 52, pp. 191-195.

[9] Miller S. S., Mocanu P. T. Differential Subordinations. Theory and Applications. Marcel Dekker Inc., New York, Basel, 2000.

[10] Ruscheweyh St. New criteria for univalent functions. Proc. Amet. Math. Soc., 1975, 49, pp. $109-115$.

[11] Sălăgean G. St. Subclasses of univalent functions. Lecture Notes in Math., Springer Verlag, Berlin, 1983, vol. 1013, pp. 362-372.

Received June 23, 2021.

In revised form, September 26, 2021.

Accepted September 29, 2021.

Published online October 11, 2021.

Department of Mathematics and Computer Science

University of Oradea

str. Universitatii nr. 1, 410087 Oradea, Romania

E-mail: dalb@uoradea.ro, alblupas@gmail.com 